\title{
HELEN: Using Brain Regions and Mechanisms for Story Understanding to Model Language as Human Behavior
}

\author{
Robert SWAINE \\ CTO, MindSoft Bioware / robertswaine@yahoo.com
}

\begin{abstract}
A new cognitive model is presented for large-scale representation of episodic situations and for manipulating these representations using the model's innate natural language processing mechanisms. As formulated, and implemented in part, the purpose of the model seeks to attain basic child level cognitive behavior of situational understanding. This includes general domain learning, by assigning internallyrelevant, though subjective, value to common experience (input situations), and by being taught how to analyze/synthesize component representations of those input situations. Ultimately this will allow it to learn and autonomously create situations with meaning from those components to best fit problem situations. The current model is written in $\mathrm{C}++$ as a visual interface and is implemented as a story understander with question answering and language generation capability.
\end{abstract}

\section{Introduction}

Two of the key difficulties in achieving a human-level intelligent agent are those of: first providing a flexible means for gaining common, wide-ranging human experience, and secondly applying common sense behavior and meaning for those and similar, but novel template experiences. The first problem is managed if there is a human analogous means flexible enough to represent many common human experience. The second problem is better handled if there is a model that can suitably link and implement the behavioral and meaning value mechanisms that relate to these common experience templates.

The model presented here provides one such solution, where the representation method and behavioral mechanisms are tied into one design. The model is a novel cognitive architecture called HELEN-KLR (namesake: Helen Keller, blind-deaf, Hierarchical Event-language Learning Network for Knowledge Representation), and follows along the lines of the SOAR and ACT-R cognitive models. The layout of the model is inspired by a number of global and regional circuit maps in mammalian neuroanatomy.

\section{Representation}

The HELEN model representation is based on a framework of neuronal registers that regionally index patterns of synaptic weights and activation levels, and reciprocally map input/output through intervening filter layers. The registers' content-maps can access, read, and modify the contents of other registers. Using these registers, HELEN represents portions of human "experience" in two major interconnected divisions: (1) a

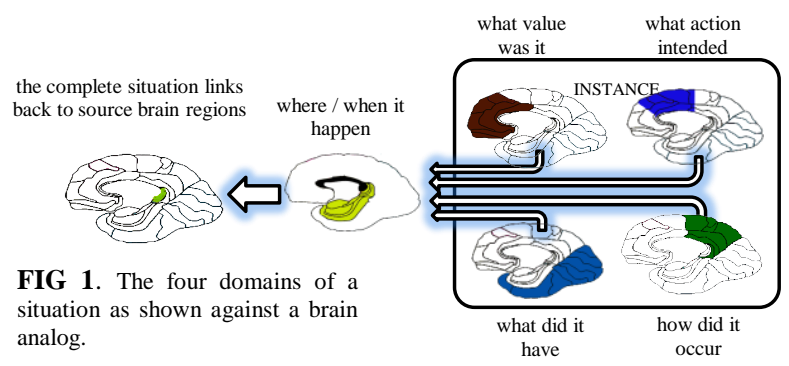

BODY, the animal's presence in a "world" environment, including the internal body state, and (2) a WORLD apart from the body that is conveyed via sensory perception. Both divisions are further subdivided into two relational domains: (A) the modeled-sensory content of the input types and (B) the relationships between any referenced content. The resulting four domains form the key representational structure of the HELEN model's version of human experience. The domains are named: BODY [Value, Role] and WORLD [Relation, Feature].

Value reflects all sensed internal body states, e.g. goal, emotional body state, valence, consequence, etc. Role represents all effort levels and patterns of directional intent for planned actions, e.g. action sets, behavioral responses, skill, functions, etc. Relation are static and dynamic arrangement patterns of attention regions relative to one region (reference or view point), e.g. motion patterns, structural layout, size, accumulated change/quantity, etc. Feature contains all sensory forms and grouped representations having some component external to the body as they are modeled, e.g., visual, tactile, olfactory, models, etc. The four domains form the mixed content of a register matrix for any objectifiable Instance by the weight pattern set by activation within the specific regions of uninhibited attention, i.e., all domains content can be made into an "object" as an instance.

Each domain is hierarchically organized along a number of sensory modalities (input types); sensors path for each domain converge and interlink at different levels of the hierarchy to form various modal and cross-modal concept models. Some sensors are dedicated to a given do-

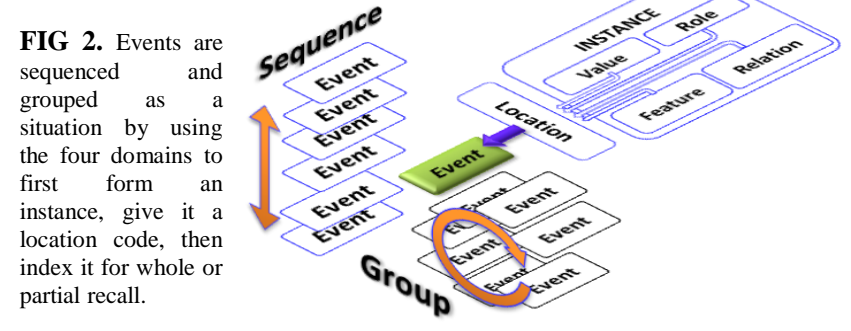


main, while others share sensor data. FIG 1. and 2 show a basic layout of the domains based on a simple brain analogue. Outside the four domains, there are two other regions in the HELEN model: Location (non view-point referenced world position) and Event.[1,2] Location is represented via changes in Relation and Feature domains, while Event is also similarly analyzed; they are both explicitly outside direct apprehension via the four domains. The model codes instances with a location, and then indexes co-occurring and co-located groups of instances as events. Events are in turn, indexed both sequentially and spatially as situations; situations are likened to episodes, or frames in some models. [3,4]

\section{Mechanisms}

In the model, mechanisms for simple and complex behavior can factor (analyze content) and manipulate (modify associated attributes of) the four domains, as well as steer the attention and focus-detail of content in situations perceived by the model so as to attach values. The structural layout of connections and the operation of the register elements comprise the major functional mechanisms, i.e., a large number of HELEN's mechanisms innately arise from it's representation method's register map. At the low-end, the predominant mechanisms include a "Long Term Potentiation" (LTP/D) decay spectrum for memories; synaptic-field copying to store activation-weight patterns; and "Combine-Compare-Contrast" circuits for reasoning and cognitive blending (situational juxtapositioning - used to synthesize or analyze). At the higher-end, dominant mechanisms include two regional indexing modules for learning and mapping meaning (values) to objects (features), and for planning, i.e., filtering then selecting actions by mapping intentions (roles) to position (relation). Two regions serve to globally link and then regionally store current and long term situation index maps. Many of the mechanisms in the model apply to its use in language understanding, i.e., giving situational meaning.

Taking a brief look at one mechanism, attention, the value system cycles between an inward and outward mode for action-response, observe-acknowledge, and store-recall modes. All situations have a salient or natural attention, a "what". Mitigated by value, natural attention serves as the "subject" of a sentence, the current "area" of a discussion, or the "what" of any event or object. These invariant or changing salient attention regions form time/space patterns that act as small/large situational borders. In the model, when taking in a situation, the size and duration of the natural attention region will also determine focus depth (detail) and focus rate used to accept the current and next situation. When HELEN is recalling a situation (externally prompted or desired), the reverse occurs: the attention region and focus detail of the recalled situations will attempt to set the attention region and focus level to their salient content, unless inhibited (internal/external focus mode).

\section{Remarks and Future Work}

At the lowest, primitive-end, HELEN can conceptualize, i.e. represent, general sensory models such as light-dark, direction, change, body-state. At the highest-end, the largest situation that HELEN conceptualizes using it's representational domains is the "game-story" concept. These are groups-sequence steps of situational intentions and moves (goals\&plans) and setssequence paths of a arranged space and character objects (setting\&content). Based on it's biological analogue, one implication is that a "story-understander / game-player" is a viable method for approaching human/mammal-like cognitive behavior: HELEN attempts to fit (make sense of) all situations which it values, into some simple/intricate event-coherent "story" or "game" model (scheme); bad fitting input raises questions in HELEN. Thus problemsolving is handled as game-story situations (rules as RoleRelation links). This is equivalent to understanding the "games" and "stories" in physics or programming or dating, when reading and summarizing the content of a physics article, laying out the execution steps in an AI program, or making sense of your child's dating troubles. Additional to these, HELEN's four domain model serves as a flexible subject-ontology generator, metaphor map, and is used to dynamically categorize novel situational inputs on-the-fly into the domains.

In parallel with completing the implementation of the model with a more detailed representation and mechanisms, the model will incorporate a large situational corpus of human-centric situations of a child in the formatives years, e.g. family and naive physics events, playing the spectrum of roles from observer to actor. Furthermore, this corpus of related/independent situations will be given most emphasis on common (cultural and language relevant) rules for manipulating (e.g. expressing) situations. As a dictionary of human childhood "experience", it will serve as the source of an initial meaning (value) template when HELEN is given situations presented in natural language as part of a novice-to-expert curriculums in various fields.

\section{References}

[1] Conjunctive representation of position, direction, and velocity in entorhinal cortex. Sargolini F, Fyhn M, Hafting T, McNaughton BL, Witter MP, Moser MB, Moser EI. Science. 2006 May 5;312(5774):680-1

[2] Place cells, grid cells, and the brain's spatial representation system. Moser EI, Kropff E, Moser MB. Annual Review of Neuroscience 2008; 31:69-89

[3] Frame Activated Inferences in a Story Understanding Program. Peter Norvig IJCAI 1983: 624-626

[4] Six Problems for Story Understanders. Peter Norvig AAAI 1983: 284-287 